\title{
WILEY-VCH
}

\section{Catalytically Active Copper Phosphate-Dextran Sulfate Microparticle Coatings for Bioanalyte Sensing}

Pietro Pacchin Tomanin, Sukhvir Kaur Bhangu, Frank Caruso* and Francesca Cavalieri*

Dr. P Pacchin Tomanin, Dr. S K Bhangu, Prof. F Caruso

ARC Centre of Excellence in Convergent Bio-Nano Science and Technology, and the Department of Chemical Engineering, The University of Melbourne, Parkville, Victoria 3010, Australia.

E-mail:fcaruso@unimelb.edu.au

Dr. S K Bhangu Dr F. Cavalieri

School of Science, RMIT University, Melbourne, Victoria 3000, Australia.

E-mail: francesca.cavalieri@rmit.edu.au

Dr F. Cavalieri.

Dipartimento di Scienze e Tecnologie Chimiche, Università di Roma "Tor Vergata", via della ricerca scientifica 1, 00133, Rome, Italy.

Keywords: porous microparticles, nonenzymatic sensors, copper phosphate, dextran sulfate

Engineering reactive and functional nanostructured surfaces is important for enhancing the sensitivity and versatility of biosensors and microreactors. For example, the assembly of hybrid inorganic-organic porous microparticles on surfaces may provide a catalytic microenvironment for a wide range of reactions. Herein, the synthesis of catalytically active porous dextran sulfate-copper phosphate hybrid microparticles by a facile and rapid crystallization process in aqueous solution is reported. The sulfated polysaccharide enabled control over the size and hierarchical morphology of the hybrid microparticles, as well as their assembly into stable macroporous coatings. The engineered microparticle coatings displayed intrinsic nonenzymatic peroxidase-like catalytic activity when employed as a platform for the detection of hydrogen peroxide. Pairing of the microparticle coating with glucose oxidase afforded a hybrid platform that was employed as a glucose sensor for monitoring physiological concentrations of a given analyte via a hybrid enzymatic/nonenzymatic cascade reaction. This work presents a strategy for the assembly of hybrid porous microparticles into enzyme-mimicking surfaces for copperbased catalysis and biochemical analyte sensing. 


\section{WILEY-VCH}

\section{Introduction}

Hybrid organic-inorganic porous microparticles have attracted growing interest in recent years owing to their desirable properties in advancing the fields of biocatalysis, ${ }^{[1]}$ sensing, ${ }^{[2]}$ bioimaging, ${ }^{[3]}$ and drug delivery ${ }^{[4]}$. They can be synthesized from a variety of organic and inorganic building blocks such as proteins, ${ }^{[5]}$ polysaccharides, ${ }^{[6]}$ enzymes, ${ }^{[7]}$ DNA, ${ }^{[8]}$ biosurfactants, ${ }^{[9]}$ and amino acids, ${ }^{[10]}$ and metal salts (e.g., $\mathrm{Cu}^{2+}, \mathrm{Ca}^{2+}, \mathrm{Mg}^{2+}, \mathrm{Mn}^{2+}$, and $\mathrm{Co}^{2+}$ ) respectively. ${ }^{[11]}$ These materials can be designed into hierarchical structures that span the nanoto-microscale with a high surface-to-volume ratio and multifunctional properties, which are useful for numerous biomedical applications. For instance, when enzymes are used, the resulting hybrid porous microparticles exhibit biocatalytic properties with high activity, durability, and stability owing to the immobilization of the enzyme molecules within the inorganic architecture. ${ }^{[1 \mathrm{a}, 12]}$ Batule et al. ${ }^{[13]}$ recently reported a highly sensitive sensor for the detection of glucose (Glu); the sensor was obtained via the sonochemical synthesis of bovine serum albumin (BSA)- and glucose oxidase (GOx)-copper phosphate porous microparticles. These BSA-Cu-GOx microparticles showed a limit of detection (LOD) of $10 \mu \mathrm{M}$ and $3.5 \mu \mathrm{M}$ for $\mathrm{H}_{2} \mathrm{O}_{2}$ and glucose, respectively. Hybrid enzyme-inorganic porous microparticles were also employed in the development of paper-based sensors for Glu detection using $\mathrm{Mn}_{3}\left(\mathrm{PO}_{4}\right)_{2}$ and GOx. ${ }^{[14]}$ The paper based sensing strips were able to detect glucose in range of $0.1-20 \mathrm{mM}$ with detection limit of $0.01 \mathrm{mM}$. However, difficulties were encountered in obtaining homogeneous and stable hierarchical microparticles (i.e., narrow size distribution, controlled porosity). Such limitations can potentially reduce the enzymatic activity of the sensors, as well as the accuracy and reproducibility of the analysis.

Hybrid organic-inorganic copper-based porous microparticles have been reported to possess

intrinsic nonenzymatic biomimetic peroxidase-like ${ }^{[10,13]}$ and oxidase-like activity. ${ }^{[15]}$ The morphology of the copper phosphate porous microparticles is influenced by the organic 


\section{WILEY-VCH}

component. For instance, Ge at al. ${ }^{[1 a]}$ demonstrated the synthesis of hybrid copper phosphateBSA porous microparticles where the $\mathrm{Cu}^{2+}-\mathrm{BSA}$ complex served as a nucleation site for the formation of primary crystals and BSA acted as a binder for the subsequent formation of hierarchical nanostructures during the growth of the porous microparticle crystals. Recently, GOx-Cu hybrid microparticle with amine-functionalized magnetic nanoparticles (MNPs) were synthesized and utilized as an effective antibacterial agent due to $\mathrm{H}_{2} \mathrm{O}_{2}$ generation ${ }^{[16]}$. In addition, Cheon et al. ${ }^{[17]}$ reported the formation of Cu-GOx-MNPs porous microparticles for the detection of glucose with high sensitivity (LOD of $2.5 \times 10^{-6} \mathrm{M}$ ) and selectivity.

Although, hybrid copper-based porous microparticles have been widely applied in suspension, $[1 a, 10,13,15]$ whereas their use in the fabrication of nanostructured catalytic surfaces remains largely unexplored. A recent study reported the fabrication of a paper-based sensor for the detection of Glu via in situ growth of copper nanoflowers on reduced graphene oxide sheets. ${ }^{[18]}$ Herein, we report the synthesis of hybrid dextran sulfate (DS) and copper phosphate porous microparticles (Cu-DS microparticles) via a simple and rapid crystallization method under mild conditions (aqueous solution, room temperature and neutral $\mathrm{pH}$ ). $\mathrm{DS}$ is chosen as it is readily available, inexpensive (as opposed to proteins, peptides, and nucleic acids), nontoxic, and biodegradable. It is also widely employed in the food, pharmaceutical, and chemical industries. ${ }^{[19]}$ Furthermore, DS is a known versatile building block for the fabrication of micronanoparticles owing to its stability over a wide range of $\mathrm{pH}$, temperature, and ionic strength. ${ }^{[20]}$ The complexing properties of DS to $\mathrm{Cu}^{2+}$ were examined with respect to controlling the size and morphology of the microparticles.

To obtain $\mathrm{Cu}$-DS porous microparticles with high a surface-to-volume ratio and optimal morphology and stability, various synthesis parameters such as $\mathrm{pH}$, DS and copper ion concentrations, and reaction time were studied. The optimized porous microparticles were subsequently used for generating functional coatings that were employed for the detection of biological analytes. These hybrid nanostructured coatings exhibited nonenzymatic peroxidase- 


\section{WILEY-VCH}

like catalytic activity and were employed as a fluorometric sensor to detect hydrogen peroxide. Furthermore, the nanostructured coatings were used in combination with GOx to detect Glu, relying on a hybrid enzymatic/nonenzymatic cascade reaction. Our results highlight the potential of $\mathrm{Cu}$-DS porous microparticles to assemble into reactive and stable nanostructured biosensing coatings for the detection of biochemical analytes.

\section{Results and discussions}

\subsection{Synthesis and characterization of hybrid Cu-DS microparticles}

Hybrid Cu-DS porous microparticles were prepared via a simple crystallization method (Figure 1a). The synthesis involved the mixing PBS, DS, and $\mathrm{CuSO}_{4}$, followed by static incubation. The morphologies and chemical composition of the $\mathrm{Cu}$-DS microparticles were comprehensively characterized by SEM, TEM, FTIR, EDX, XRD, and TGA (Figure 1b-e and Figure S1 and S2). The Cu-DS microparticles had a diameter of $3.0 \pm 0.1 \mu \mathrm{m}$ and a homogeneous size distribution, as shown in the SEM (Figure 1b) and TEM (Figure 1c) images. The hierarchical architecture and morphology of the Cu-DS microparticles were ascribed to the continuous nucleation and growth processes that resulted in interconnected nanometer-thick sheets (Figure 1b). Furthermore, TEM images revealed that the core of the Cu-DS microparticles was denser than their surface (Figure 1c, inset).

The FTIR spectrum of the $\mathrm{Cu}-\mathrm{DS}$ microparticles indicated the presence of $\mathrm{P}-\mathrm{O}$ and $\mathrm{P}=\mathrm{O}$ owing to the presence of strong peaks at $390,560,625,990$ and $1035 \mathrm{~cm}^{-1}$, which can be assigned to phosphate groups (Figure 1d). ${ }^{17}$ In addition, the bands in the regions from 1180 to $1300 \mathrm{~cm}^{-1}$ and 680 to $870 \mathrm{~cm}^{-1}$ were ascribed to vibrations related to the sulfate groups in the dextran chain, thus confirming the presence of DS in the hybrid microparticles. ${ }^{18}$ This was further confirmed by EDX spectroscopy and elemental mapping, which revealed the presence of $\mathrm{P}, \mathrm{Cu}$, $\mathrm{O}$, and $\mathrm{S}$ elements in the $\mathrm{Cu}-\mathrm{DS}$ microparticles (Figure 1e). Furthermore, as noted from elemental mapping, DS appeared to be evenly distributed over the surface of the Cu-DS 


\section{WILEY-VCH}

microparticles. The XRD spectrum of the $\mathrm{Cu}$-DS microparticles displayed sharp diffraction peaks within an amorphous background (Figure S1). The position and relative intensities of the peaks matched well with those of pure copper phosphate hydrate crystals (PDF \#22-0548). The broad peak centered at $20^{\circ}$ was attributed to the presence of amorphous DS in the microparticles. ${ }^{19}$ These findings suggest that the hybrid microparticles were composed of amorphous DS chains embedded within well-crystalline $\mathrm{Cu}_{3}\left(\mathrm{PO}_{4}\right)_{2} \cdot 3 \mathrm{H}_{2} \mathrm{O}$ nanosheets.

To quantify the weight percentage of DS in the Cu-DS microparticles, TGA was performed (Figure S2). A weight loss of $8 \%$ was observed upon heating the $\mathrm{Cu}$-DS microparticles up to $100{ }^{\circ} \mathrm{C}$, which was attributed to water desorption. The subsequent weight loss of $6 \%$, with further heating up to $300^{\circ} \mathrm{C}$, was likely due to coordinated water molecules in $\mathrm{Cu}_{3}\left(\mathrm{PO}_{4}\right)_{2}$. The additional weight loss of $6 \%$ observed between 300 and $440{ }^{\circ} \mathrm{C}$ was attributed to the decomposition of DS. ${ }^{21}$ From 440 to $600{ }^{\circ} \mathrm{C}$, no further weight loss was observed, indicating that combustion of the organic component was complete. These results further confirmed the presence of DS within the nanostructured hybrid Cu-DS microparticles.

\subsection{Tailoring the surface morphology, size distribution and stability of Cu-DS microparticles}

To gain an insight into the formation mechanism, stability and to optimize the size distribution and porosity of the $\mathrm{Cu}$-DS microparticles, the effects of DS, copper sulfate, PBS concentrations and $\mathrm{pH}$ on the structural characteristics of the $\mathrm{Cu}-\mathrm{DS}$ microparticles were investigated.

Similar to conventional crystallization processes, the formation of porous hybrid $\mathrm{Cu}-\mathrm{DS}$ microparticles proceeded through nucleation and growth of nuclei. ${ }^{[1 a]}$ Furthermore, in hybrid microparticles, the organic component plays an active role on the interconnection of the growing crystalline nanosheets. Herein, we hypothesize that DS might play an essential role in promoting and controlling the nucleation and growth of the crystalline nanosheets, and thus can act as a binder for the assembly of the nanosheets into hierarchical structures. After incubation of $\mathrm{DS}, \mathrm{CuSO}_{4}$ and $\mathrm{PBS}$ for $30 \mathrm{~min}$, the formation of microparticles with a few nanosheets 


\section{WILEY-VCH}

exposed on the surface was observed (Figure 2a). With increasing incubation times (60 and 90 min), the kinetically controlled growth of numerous crystalline nanosheets occurred, resembling petals on a flower-like structure (Figure $\mathbf{2 b}$ and $\mathbf{c}$ ). At prolonged incubation time (180 min), the anisotropic growth of the petals was complete, leading to the formation of spherical Cu-DS microparticles with a high nanosheet (petal-like) density (Figure 2d). The interaction between $\mathrm{DS}$ and $\mathrm{Cu}_{3}\left(\mathrm{PO}_{4}\right)_{2}$ crystals is likely driven by the electrostatic complexation between the sulfate groups and $\mathrm{Cu}^{2+}$ ions. We note that interactions between $\mathrm{Cu}^{2+}$ and $\mathrm{OH}$ groups of DS cannot be excluded.

To confirm and understand the role of DS on the crystallization process of the hybrid microparticles, a control experiment was performed. Pure copper phosphate particles were prepared in the absence of DS. Pure copper phosphate microparticles formed after incubation for 180 min (Figures $\mathbf{S 3}$ and $\mathbf{S 4 b})$, were larger $(7.0 \pm 2.5 \mu \mathrm{m})$ and polydisperse, with a higher tendency to aggregate when compared with the $\mathrm{Cu}$-DS particles. These observations suggest that DS not only provides a scaffold for the crystallization and arrangement of nanosheets in highly porous, monodisperse, and stable Cu-DS microparticles but also prevents the aggregation of the microparticles, hence acting as a stabilizer.

To further optimize the size distribution, porosity and structural stability of the Cu-DS microparticles and enable their use as biosensors, various experimental parameters including the concentration of reagents, concentrations of salts, and $\mathrm{pH}$, were varied. The effect of the concentration of PBS was investigated by preparing Cu-DS microparticles at different PBS concentrations (1-100 mM). At low PBS salt concentrations of 1 and $2 \mathrm{mM}$, no precipitates or particles were obtained. In contrast, well-defined particles with increasing diameters from 2.8 \pm 0.2 to $4.3 \pm 0.3 \mu \mathrm{m}$ and with similar surface morphologies were achieved at higher PBS salt concentrations of $5 \mathrm{mM}$ (Figure S5a), $10 \mathrm{mM}$ (Figure 1b), and $20 \mathrm{mM}$ (Figure S5b), respectively. At higher PBS salt concentrations of 50 and $100 \mathrm{mM}$, crystal-like aggregates, instead of particles, were formed (Figure S5c and d). These results suggest that at low 


\section{WILEY-VCH}

concentrations of phosphate ions, the concentration of $\mathrm{Cu}_{3}\left(\mathrm{PO}_{4}\right)$ is insufficiently low to initiate nucleation for microparticle formation. Conversely, high concentrations of phosphate ions prevent the crystals from organizing into ordered hierarchical nanostructures owing to the rapid nucleation and growth of the copper phosphate crystals.

The effect of the copper ion concentration $(2.5-160 \mathrm{mM})$ on the synthesis of the $\mathrm{Cu}$-DS microparticles was also examined (Figure 3a). At the lowest concentration of copper ions of $2.5 \mathrm{mM}$, the particles featured micrometer-sized pores and sparingly distributed petals on the surface (Figure 3a1). When higher concentrations of copper ions were used (5-160 mM), the degree of compactness of the petals formed on the surface of the Cu-DS microparticles (Figure 3a2-4) increased, resulting in a smaller petal-to-petal distance. In addition, the Cu-DS microparticles obtained at lower concentrations of copper ions ( 2.5 and $5 \mathrm{mM})$ were larger $(\sim 6-$ $8 \mu \mathrm{m})$ than the $\mathrm{Cu}$-DS particles $(\sim 3-4 \mu \mathrm{m})$ obtained at higher concentrations of copper ions (40 and $160 \mathrm{mM})$. The size of the particles was likely correlated to the number of nucleation sites, which is determined by the concentration of the copper ions available during the crystallization process.

The effect of $\mathrm{pH}(5.4-10.4)$ on the morphology and stability of the $\mathrm{Cu}$-DS microparticles was subsequently examined (Figure 3b). The Cu-DS microparticles obtained at $\mathrm{pH}$ 5.4, when phosphate ions are protonated, showed an incomplete structure with a low petal density $(\mathrm{pH}$ 5.4, Figure 3b1) or lack of formation $(\mathrm{pH}<5.4)$. Conversely, the $\mathrm{Cu}-\mathrm{DS}$ microparticles synthesized at higher $\mathrm{pH}$ values from 6.4 to 10.4 were well dispersed, stable, and featured comparable sizes and petals on the surface (Figure 3b2-4 and Figure 1b).

In addition, the morphology of the $\mathrm{Cu}$-DS microparticles prepared at varying DS concentrations from 0.08 to $8.3 \mathrm{mg} \mathrm{mL}^{-1}$ was studied (Figures 3c1-4 and S6). Aggregated and poorly organized structures were observed at the lower DS concentration $\left(0.08-0.17 \mathrm{mg} \mathrm{mL}^{-1}\right)$ (Figure 3c1 and $\mathbf{c 2}$ ) when compared with the more uniform and well-dispersed flower-like microparticles formed at the higher DS concentrations $\left(1.7-8.3 \mathrm{mg} \mathrm{mL}^{-1}\right.$ ) (Figure $3 \mathbf{c} 3$ and $\mathbf{c 4}$ 


\section{WILEY-VCH}

and S6). Overall, these results suggest that the concentration of reagents and $\mathrm{pH}$ are important parameters for controlling the morphology of the microparticles.

\subsection{In situ formation of catalytically active $\mathrm{Cu}$-DS microparticle coatings}

As the crystallization process for the synthesis of hybrid microparticles proceeds via interactions between metal ions and functional moieties, such as sulfate, hydroxyl and carboxyl groups, the in-situ formation of the $\mathrm{Cu}-\mathrm{DS}$ microparticles and their assembly into functional coatings was examined using multi-well tissue culture (TC)-treated polystyrene microplates (PS-plates). The PS-plates feature negatively charged carboxyl and hydroxyl functional groups on the surface that are typically exposed to the cell culture medium. ${ }^{[21]}$ These surface groups likely act as nucleation sites for the formation of primary $\mathrm{Cu}_{3}\left(\mathrm{PO}_{4}\right)$ crystals, thereby enabling coating of the surface with microparticles. The nanostructured (coated) PS-plates were prepared by adding aliquots of a solution of DS in $\mathrm{PBS}\left(\mathrm{pH}\right.$ 7.4) mixed with $\mathrm{CuSO}_{4}$ solution into each well of a 96-well TC plate, followed by incubation at $21{ }^{\circ} \mathrm{C}$ for $16 \mathrm{~h}$. The coating obtained at the base of the wells, as indicated by an opaque blue layer, was analyzed by SEM after the clear supernatant was discarded. The SEM images in Figure 4 revealed a micro-to-nano hierarchical homogeneous coating (on the well surface), suggesting the strong interaction between the microparticles and the surface as well as among the microparticles Furthermore, the coating was composed of $\mathrm{Cu}-\mathrm{DS}$ microparticles featuring similar morphologies to those obtained without the use of the PS-plates. The structural integrity and stability of the coating was confirmed by optical microscopy inspection of surfaces after several days of soaking in water. The strong interaction between the $\mathrm{Cu}$-DS microparticles and PS-plates can be attributed to the presence of hydrophilic chemical groups on the plate surface (mostly $\mathrm{OH}$ and $\mathrm{COOH}$ groups) that can interact with $\mathrm{Cu}^{2+}$ to generate nucleation sites for crystal growth and subsequent formation of layers of Cu-DS microparticles interconnected by DS chains. This hypothesis was supported by the fact that the Cu-DS microparticles did not adhere on the PS surface of the vials that lacked hydrophilic functional groups. The stable macroporous structure of the Cu-DS 


\section{WILEY-VCH}

microparticle coating is desirable for effective mass transport to the solid-liquid interface and catalytic reactivity.

\subsection{High-throughput detection and sensing of $\mathrm{H}_{2} \mathrm{O}_{2}$ and Glu}

The $\mathrm{Cu}$-DS microparticle coatings were used as reactors for chemical reactions to develop a high-throughput detection and sensing platform. The platform was first examined for the detection of hydrogen peroxide (Figure 5a). Specifically, the nonenzymatic peroxidase-like catalytic activity of the Cu-DS microparticle coating was studied using OPD as the reactant (Figure 5a), which is oxidized by $\mathrm{H}_{2} \mathrm{O}_{2}$ to fluorescent 2,3-diaminophenazine (DAP) via a condensation reaction. DAP has an absorbance peak at $410 \mathrm{~nm}$ and an emission peak at $566 \mathrm{~nm}$ at $21{ }^{\circ} \mathrm{C}$, which can be used to monitor the reaction in real time (i.e., reaction kinetics). Fluorescence measurements were performed over absorbance measurements to avoid the potential contribution of scattering from the $\mathrm{Cu}$-DS microparticles immobilized on the well surface of the TC-treated PS plates. To verify this, the fluorescence emission intensities of DAP solution in non-coated and $\mathrm{Cu}-\mathrm{DS}$ microparticle-coated wells were recorded. The intensities were comparable, within the standard deviation, demonstrating that the fluorescence intensity profile of the DAP solution is not influenced by the presence of the $\mathrm{Cu}$-DS microparticle coating at the base of the well. When a PBS solution containing OPD and $\mathrm{H}_{2} \mathrm{O}_{2}$ was placed in a Cu-DS microparticle-coated well, the emission intensity at $566 \mathrm{~nm}$ increased as a function of time and reached a plateau after 30 min of reaction (Figure 5b). Conversely, $\mathrm{H}_{2} \mathrm{O}_{2}$ could not oxidize OPD in the absence of the Cu-DS microparticle coating, and the presence of the Cu-DS microparticle coating only slightly catalyzed the condensation reaction mediated by dissolved oxygen and copper ions (Figure 5b). ${ }^{[22]}$

The rapid and efficient conversion of OPD to DAP (Figure 5b) demonstrates the nonenzymatic peroxidase-like catalytic activity of the nanostructured surface toward hydrogen peroxide as the substrate. The changes in the fluorescence intensity profile observed in Figure $\mathbf{5 b}$ were used to 


\section{WILEY-VCH}

determine the rate law and rate for the condensation reaction. The conversion of OPD into DAP followed a pseudo-first-order reaction, as observed from the linear trends of the natural logarithm of the fluorescence emission intensity against time $\left(R^{2}>0.99\right)$ (Figure 5c). Different rate constants were obtained depending on the presence of the $\mathrm{Cu}$-DS microparticle coating and/or $\mathrm{H}_{2} \mathrm{O}_{2}: 0.152 \pm 0.002,4.36 \pm 0.07$, and $122.2 \pm 0.4 \mathrm{~ms}^{-1}$ when OPD was incubated with $\mathrm{H}_{2} \mathrm{O}_{2}$ only, the $\mathrm{Cu}-\mathrm{DS}$ microparticle coating only, or with $\mathrm{H}_{2} \mathrm{O}_{2}$ and the $\mathrm{Cu}-\mathrm{DS}$ microparticle coating, respectively. The high catalytic activity of the Cu-DS microparticles could be attributed to their porous morphology and chemical composition, i.e., high surface-to-volume ratio and high accessibility to the copper ion active sites on the surface. This was also confirmed by preparing $\mathrm{Cu}$-DS microparticles coatings with different morphologies at $\mathrm{pH} 5.4$ (less porous structure - Figure 3b1) and pH 7.4 (highly porous structure - Figure 4) for the detection of $\mathrm{H}_{2} \mathrm{O}_{2}$. The coatings prepared at $\mathrm{pH} 7.4$ showed a more efficient peroxidase-like catalytic activity (Figure S 7b) and faster kinetics (Figure S7c) than coatings obtained at pH 5.4, due to a large surface-to-volume ratio (Figure S7).

As the concentration of DAP can be directly correlated to the amount of $\mathrm{OH}$ radicals as well as $\mathrm{H}_{2} \mathrm{O}_{2}$, the engineered microporous platform was used for $\mathrm{H}_{2} \mathrm{O}_{2}$ sensing. To confirm the quantitative detection of $\mathrm{H}_{2} \mathrm{O}_{2}$, PBS solutions ( $\mathrm{pH}$ 6.5) containing different concentrations of $\mathrm{H}_{2} \mathrm{O}_{2}$ (from 0 to $7 \mathrm{mM}$ ) and OPD $(0.1 \mathrm{mM})$ were added to the $\mathrm{Cu}$-DS microparticle-coated wells and kept under gentle shaking for $45 \mathrm{~min}$ at $21{ }^{\circ} \mathrm{C}$ (Figure 5d). With an increase in $\mathrm{H}_{2} \mathrm{O}_{2}$ concentration, the color of the reaction solutions gradually changed from colorless to dark yellow, which coincided with an increase in the intensity of the fluorescence emission peak at $566 \mathrm{~nm}$ (Figure 5e). A linear trend $\left(R^{2}=0.996\right)$ was observed within a $\mathrm{H}_{2} \mathrm{O}_{2}$ concentration range of $0-0.4 \mathrm{mM}$ with a sensitivity of $28.3 \pm 0.6 \mathrm{kcps} \mathrm{mM}^{-1}$ and a limit of detection (LOD) of $35 \mu \mathrm{M}$ (signal-to-noise $(\mathrm{S} / \mathrm{N})$ ratio $=3$ ). At the higher range of $\mathrm{H}_{2} \mathrm{O}_{2}$ concentrations of $0.4-$ $7 \mathrm{mM}$, a nonlinear Langmuir-like fit was observed (Table S1). Importantly, the Cu-DS microparticle coating was stable during the reaction and unbound particles were not detected in 


\section{WILEY-VCH}

the supernatant after completion of the reaction, indicating good mechanical stability of the coating. Overall, the findings demonstrate that the present engineered platform enables the detection of $\mathrm{H}_{2} \mathrm{O}_{2}$ in the millimolar concentration range, which corresponds to the concentrations of many bio-analytes present in biological fluids such as Glu. ${ }^{[23]}$

By pairing this nonenzymatic sensing platform to a $\mathrm{H}_{2} \mathrm{O}_{2}$-producing enzyme such as $\mathrm{GOx}$ a versatile detection platform for the detection and sensing of Glu was engineered. Specifically, we combined the enzymatic activity of GOx and the nonenzymatic peroxidase-like catalytic activity of the present platform to detect Glu via a hybrid enzymatic/nonenzymatic cascade reaction (Figure 6a). The cascade reaction mechanism of the hybrid platform consists of (i) a GOx-mediated enzymatic reaction, where Glu and oxygen react to form gluconolactone and $\mathrm{H}_{2} \mathrm{O}_{2}$ and the concentration of $\mathrm{H}_{2} \mathrm{O}_{2}$ can be directly correlated to the amount of $\mathrm{Glu}$ (as one equivalent of Glu produces one equivalent of $\mathrm{H}_{2} \mathrm{O}_{2}$ ) and (ii) a nonenzymatic reaction, where hydroxyl radicals are produced from $\mathrm{H}_{2} \mathrm{O}_{2}$ on the $\mathrm{Cu}$-DS microparticle catalytically active coating to promote the condensation reaction of OPD to DAP. This cascade reaction would result in changes in the color of the solution and fluorescence properties that can be measured by a fluorometric method. To assess the occurrence of a hybrid cascade reaction, a solution of OPD, GOx, and Glu in PBS was incubated at $37^{\circ} \mathrm{C}$ in the presence of the $\mathrm{Cu}-\mathrm{DS}$ microparticle coating, and the emission and excitation spectra of that solution were recorded (Figure $6 \mathbf{b}$, black curve). For the control experiments, two solutions lacking the cascade reaction components were prepared in the absence of the Cu-DS microparticle coating or Glu (Figure $\mathbf{6 b}$, green and red curves, respectively). When all the reaction components were present, a significant increase of both the excitation and emission peak intensities was observed (Figure 6b), indicating the effective production of DAP. Conversely, the weaker fluorescence intensities displayed by both control solutions indicate the ineffective production of DAP (Figure 6b). Notably, when OPD, GOx, and Glu in PBS were incubated in the presence of the $\mathrm{Cu}-\mathrm{DS}$ microparticle coating at different concentrations of Glu, the emission peak intensity at 


\section{WILEY-VCH}

$536 \mathrm{~nm}$ increased as a function of the initial concentration of Glu (Figure 6c). A linear trend $\left(R^{2}=0.976\right)$ was observed in the Glu concentration range of $0-1 \mathrm{mM}$ with a sensitivity of 1180 $\pm 70 \mathrm{kcps} \mathrm{mM}^{-1}$ and an LOD of $35 \mu \mathrm{M}(\mathrm{S} / \mathrm{N}=3)$. A nonlinear Langmuir-like fit was observed for concentrations beyond this range (Table S2). It is worth noting that the LOD and detection range of the engineered sensing system are within the physiological concentration range of Glu in healthy and diabetic patients $(1-30 \mathrm{mM}) .{ }^{[24]}$. In addition, to test the selectivity of system towards glucose, the emission intensity of DAP was studied after the addition of other sugars such as lactose $(1.5 \mu \mathrm{M})$, fructose $(0.1 \mathrm{mM})$, galactose $(3 \mu \mathrm{M})$. Figure $\mathbf{S 8}$ shows an increase in emission intensity as a function of time, when $\mathrm{Cu}-\mathrm{DS}$ microparticle were incubated with 1.5 mM Glu, OPD, GOx, in PBS. However, negligible changes in the emission intensity were observed in the presence of other sugars suggesting the selectivity of the $\mathrm{Cu}$-DS microparticles for accurate determination of Glu.

\section{Conclusions}

We have reported the synthesis of dextran sulfate-copper phosphate hybrid microparticles via a simple, rapid, and low-cost crystallization method without requiring harsh reaction conditions such as high temperature, high pressure, and organic solvents. The synthesis parameters were investigated and optimized to prepare monodisperse particles with a high surface-to-volume ratio, hierarchical surface morphology, and catalytically active sites. Dextran sulfate, as an organic component, played a key role in tuning the size, morphology and stability of the hybrid microparticles. The synthesis strategy was applied to tissue culture-treated polystyrene plates for the facile and scalable preparation of $\mathrm{Cu}$-DS microparticle coatings in situ. The resulting coating platform afforded high-throughput detection and sensing of $\mathrm{H}_{2} \mathrm{O}_{2}$ and glucose in PBS at physiological concentrations with high sensitivity. We envision that this system can be exploited to detect other biological analytes, such as cholesterol, lactic acid, and uric acid, when 


\section{WILEY-VCH}

used in combination with cholesterol oxidase, lactate oxidase, and urate oxidase, respectively, upon the enzyme-mediated production of $\mathrm{H}_{2} \mathrm{O}_{2}$.

\section{Experimental section}

\section{Materials}

Copper sulfate, phosphate-buffered saline (PBS) tablets, dextran sulfate sodium salt from Leuconostoc spp. $\left(M_{\mathrm{w}}>500 \mathrm{kDa}\right)$, dextran from Leuconostoc spp. $\left(M_{\mathrm{w}}=450-650 \mathrm{kDa}\right)$, hydrochloric acid, D-(+)-Galactose, fructose, lactose, sodium hydroxide, hydrogen peroxide, $\mathrm{GO}_{\mathrm{x}}$ from Aspergillus niger, Corning 96-well tissue culture (TC)-treated polystyrene plates were purchased from Sigma-Aldrich, o-phenylenediamine (OPD) was purchased from Alfa Aesar and Glu was purchased from AnalaR. All the chemicals were used as received. Highpurity (Milli-Q) water with a resistivity of $18.2 \mathrm{M} \Omega \mathrm{cm}$ was obtained from an inline Millipore RiOs/origin water purification system.

\section{Synthesis of hybrid Cu-DS porous microparticles}

In a typical synthesis, $500 \mu \mathrm{L}$ of PBS (10 mM, pH 7.4) with a DS concentration of $1 \mathrm{mg} \mathrm{mL}^{-1}$ was quickly added under vortex mixing to $100 \mu \mathrm{L}$ of a $\mathrm{CuSO}_{4}$ aqueous solution $(120 \mathrm{mM})$ in a $2.2 \mathrm{~mL}$ polystyrene (PS) vial. The resulting solution was vortexed for $60 \mathrm{~s}$ and subsequently incubated under static conditions overnight at room temperature $\left(25^{\circ} \mathrm{C}\right)$. Within $30 \mathrm{~min}$ of incubation, the solution turned from transparent to opaque, indicating the nucleation and growth of micrometer-sized particles. After overnight incubation, the blue precipitate was washed via centrifugation ( $2000 \mathrm{~g}, 3 \mathrm{~min}$ ) and re-suspended in water for at least five times, then dried under vacuum at room temperature. To study the effects of the reaction synthesis parameters, the particle synthesis was performed by systematically changing one parameter at a time, namely incubation time (30-180 min), $\mathrm{pH}(5.4-10.4)$, concentrations of DS (0.08-4.2 mg/ml), $\mathrm{CuSO}_{4}$ (2.5-160 mM) and PBS (1-100 mM) A $10 \mathrm{mM}$ PBS solution constitutes $137 \mathrm{mM} \mathrm{NaCl}, 10 \mathrm{mM}$ $\mathrm{Na}_{2} \mathrm{HPO}_{4} / \mathrm{NaH}_{2} \mathrm{PO}_{4}$, and $2.7 \mathrm{mM} \mathrm{KCl}$ at $\mathrm{pH}$ 7.4.

Characterization of hybrid $C u$-DS porous microparticles 


\section{WILEY-VCH}

Transmission electron microscopy (TEM) images were acquired using a Tecnai Spirit (FEI, USA) instrument with an operating voltage of $120 \mathrm{kV}$. Scanning electron microscopy (SEM) images and energy-dispersive X-ray spectroscopy (EDX) patterns were acquired with a FlexSEM 1000 (Hitachi, Japan) benchtop scanning electron microscope equipped with a Quantax 80 (Bruker, USA) EDX system. Typically, images and spectra of uncoated samples were recorded using a beam voltage of $15 \mathrm{kV}$, operating in SEM pressure mode and with an acquisition time of $5 \mathrm{~min}$. Attenuated total reflectance Fourier transform infrared (ATR-FTIR) spectra were obtained on a TENSOR II (Bruker) ATR-FTIR spectrometer and analyzed using the software OPUS 7.8. The number of scans were 64 and the minimum resolution was $4 \mathrm{~cm}^{-1}$. Fluorescence emission and excitation spectra were recorded on an Infinite M200 microplate reader (Tecan, Switzerland) and analyzed using the software i-control. X-ray diffraction (XRD) analysis was performed on a Bruker D8 Advance X-ray diffractometer with Ni-filtered $\mathrm{Cu} \mathrm{K} \alpha$ radiation $(1.54 \AA)$. The data were collected between $2 \theta 5^{\circ}$ and $85^{\circ}$, with a step size of $0.02^{\circ}$ and a scan rate of $1.0 \mathrm{~s}$ per step. An anti-scatter blade was used to reduce the diffracted background intensity at low angles. An incident beam divergence of $1.0^{\circ}$ was used with a $2.5^{\circ}$ soller slit in the diffracted beam. The sample was spun at 15 revolutions per minute. For sample preparation, the samples were ground in a mortar and pestle in ethanol, smeared onto a glass slide, and allowed to dry overnight in air. Phase identification was performed using Diffrac. EVA V4.1 software with the ICDD PDF4+ 2018 database. Thermal gravimetric analysis (TGA) was performed on a TGA Netzsch TG 209F1 Libra instrument. The samples were heated in alumina crucibles at a heating rate of $4{ }^{\circ} \mathrm{C} \min ^{-1}$ from 30 to $600{ }^{\circ} \mathrm{C}$ in air.

Assembly of Cu-DS porous microparticles onto surfaces

To prepare a 96-well plate coated with $\mathrm{Cu}-\mathrm{DS}$ microparticles, the microparticles were formed and assembled in situ into microparticle coatings. A similar synthesis procedure to that described for the preparation of the Cu-DS microparticles was used. In a $15 \mathrm{~mL}$ PS vial, $5 \mathrm{~mL}$ of PBS (10 mM, pH 7.4 or 5.4 (where specified)) with a DS concentration of $1 \mathrm{mg} \mathrm{mL}^{-1}$ was 


\section{WILEY-VCH}

quickly added under vortex mixing to $1 \mathrm{~mL}$ of a $\mathrm{CuSO}_{4}$ aqueous solution $(240 \mathrm{mM})$. The resulting solution was vortexed for $60 \mathrm{~s}$. Aliquots $(300 \mu \mathrm{L})$ were then added to each well of a 96-well plate and subsequently incubated under static conditions overnight at room temperature. The functionalized wells were rinsed with water and stored under water at room temperature until further use.

\section{Catalytic activity and $\mathrm{H}_{2} \mathrm{O}_{2}$ and Glu sensing}

The nonenzymatic peroxidase-like activity of the hybrid $\mathrm{Cu}$-DS microparticle coatings was studied using OPD as substrate. In each Cu-DS microparticle-coated well, $100 \mu \mathrm{L}$ of OPD solution $(0.3 \mathrm{mM}), 100 \mu \mathrm{L}$ of PBS $(10 \mathrm{mM}, \mathrm{pH} 6.5)$, and $100 \mu \mathrm{L}$ of $\mathrm{H}_{2} \mathrm{O}_{2}(15 \mathrm{mM})$ were incubated under gentle mixing at $21^{\circ} \mathrm{C}$. The fluorescence emission intensity of the solution in each well was recorded in real-time every 5 min. Fluorescence emission intensities at $566 \mathrm{~nm}$ were recorded at an excitation wavelength of $410 \mathrm{~nm}$. Separation of the $\mathrm{Cu}-\mathrm{DS}$ microparticles from the solution was not required as the catalytic substrate (microparticle coating) remained attached to the base of the well during the reaction.

$\mathrm{H}_{2} \mathrm{O}_{2}$ detection and sensing was performed using the same experimental conditions as described before varying the final $\mathrm{H}_{2} \mathrm{O}_{2}$ concentration from 0 to $7 \mathrm{mM}$. For the control experiments, solutions composed of (i) OPD in PBS in the presence of Cu-DS microparticles only and (ii) OPD and $\mathrm{H}_{2} \mathrm{O}_{2}$ in PBS in the absence of $\mathrm{Cu}$-DS microparticles were incubated as previously described and their fluorescence emissions were recorded over time.

The detection and sensing of Glu was also examined in the Cu-DS microparticle-coated wells. In each well, $100 \mu \mathrm{L}$ of OPD solution $(0.3 \mathrm{mM}), 100 \mu \mathrm{L}$ of PBS (10 mM, pH 6.5) with different concentrations of Glu, and $100 \mu \mathrm{L}$ of $\mathrm{GOx}\left(100 \mathrm{U} \mathrm{mL}^{-1}\right)$ were incubated under gentle mixing at $37^{\circ} \mathrm{C}$. After $120 \mathrm{~min}$ of incubation, the solution fluorescence emission intensities at $536 \mathrm{~nm}$ were recorded at an excitation wavelength of $444 \mathrm{~nm}$.

In addition, selectivity towards Glu detection was monitored in the $\mathrm{Cu}$-DS microparticle-coated wells using lactose $(1.5 \mu \mathrm{M})$, fructose $(0.1 \mathrm{mM})$, galactose $(3 \mu \mathrm{M})$. In Cu-DS microparticle- 


\section{WILEY-VCH}

coated wells, $100 \mu \mathrm{L}$ of OPD solution $(0.3 \mathrm{mM}), 100 \mu \mathrm{L}$ of GOx $\left(100 \mathrm{U} \mathrm{mL}^{-1}\right)$ and $100 \mu \mathrm{L}$ of PBS (10 mM, pH 7.4) with Glu (final concentration $1.5 \mathrm{mM}$ ) in absence and presence of other sugars, was added under gentle mixing at $37^{\circ} \mathrm{C}$. The fluorescence emission intensities at 566 $\mathrm{nm}$ were recorded at an excitation wavelength of $410 \mathrm{~nm}$ as a function of time.

\section{Acknowledgments}

This research was conducted and funded by the Australian Research Council Centre of Excellence in Convergent Bio-Nano Science and Technology (project number CE140100036).

F. Caruso acknowledges the award of a National Health and Medical Research Council Senior Principal Research Fellowship (GNT1135806) and F.Cavalieri acknowledges the award of RMIT Vice Chancellor Senior Research Fellowship. This work was performed in part at the Materials Characterization and Fabrication Platform (MCFP) at The University of Melbourne. The authors acknowledge Dr. Maryline Chee Kimling for helpful discussions.

\section{Conflict of Interest:}

The authors declare no conflict of interest.

\section{Supporting Information.}

Supporting Information is available from the Wiley Online Library or from the author

\section{Author Contributions}

${ }^{ \pm}$P.P.T. and S.K.B. contributed equally to this work.

\section{References}

[1] a) J. Ge, J. Lei, R. N. Zare, Nat. nanotech. 2012, 7, 428; b) Z. Lin, Y. Xiao, L. Wang, Y. Yin, J. Zheng, H. Yang, G. Chen, RSC Adv. 2014, 4, 13888.

[2] J. Sun, J. Ge, W. Liu, M. Lan, H. Zhang, P. Wang, Y. Wang, Z. Niu, Nanoscale 2014, $6,255$.

[3] R. Hu, X. Zhang, Z. Zhao, G. Zhu, T. Chen, T. Fu, W. Tan, Angew. Chem. 2014, 53, 5821.

[4] X. T. Zheng, H. L. He, C. M. Li, RSC Adv. 2013, 3, 24853.

[5] a) T. Wei, D. Du, M.-J. Zhu, Y. Lin, Z. Dai, ACS Appl. Mater. Interfaces 2016, 8, 6329; b) Q. F. Li, L. X. Zeng, J. C. Wang, D. P. Tang, B. Q. Liu, G. N. Chen, M. D. Wei, $A C S$ Appl. Mater. Interfaces 2012, 4, 490.

[6] J. R. Lakkakula, T. Matshaya, R. W. M. Krause, Mater. Sci. Eng. C-Mat. 2017, 70, 169.

[7] J. Sun, J. Ge, W. Liu, M. Lan, H. Zhang, P. Wang, Y. Wang, Z. J. N. Niu, Nanoscale 2014, 6, 255. 


\section{WILEY-VCH}

[8] G. Zhu, R. Hu, Z. Zhao, Z. Chen, X. Zhang, W. Tan, J. Am. Chem. Soc. 2013, 135, 16438.

[9] J. Jiao, X. Xin, X. Wang, Z. Xie, C. Xia, W. Pan, RSC Adv. 2017, 7, 43474.

[10] Z. F. Wu, Z. Wang, Y. Zhang, Y. L. Ma, C. Y. He, H. Li, L. Chen, Q. S. Huo, L. Wang, Z. Q. Li, Sci. Rep. 2016, 6, 22412.

[11] a) S. W. Lee, S. A. Cheon, M. I. Kim, T. J. Park, J. Nanobiotechnology 2015, 13, 54; b) P. Shende, P. Kasture, R. S. Gaud, Artif. Cells Nanomed. Biotechnol. 2018, 46, 413; c) T. D. Tran, M. I. Kim, Biochip Journal 2018, 12, 268.

[12] S. K. Patel, R. K. Gupta, V. Kumar, P. Mardina, R. Lestari, V. C. Kalia, M.-S. Choi, J.K. Lee, Indian J. Microbiol. 2019, 59, 370.

[13] B. S. Batule, K. S. Park, S. Gautam, H. J. Cheon, M. I. Kim, H. G. Park, Sensor Actuat. B-Chem. 2019, 283, 749.

[14] W. Li, S. Lu, S. Bao, Z. Shi, Z. Lu, C. Li, L. Yu, Biosens. Bioelectron. 2018, 99, 603.

[15] C. Altinkaynak, E. Kocazorbaz, N. Ozdemir, F. Zihnioglu, Int. J. Biol. Macromol. 2018, 109, 205.

[16] I. Lee, H. J. Cheon, M. D. Adhikari, T. D. Tran, K.-M. Yeon, M. I. Kim, J. Kim, Int. J. Biol. Macromol. 2020, 155, 1520.

[17] H. J. Cheon, M. D. Adhikari, M. Chung, T. D. Tran, J. Kim, M. I. Kim, Adv. Healthc. Mater. 2019, 8, e1801507.

[18] B. Wang, Y. Wu, Y. Chen, B. Weng, C. Li, Sens. Actuators B Chem. 2017, 238, 802.

[19] A. L. Bhavani, J. Nisha, Int. J. Pharm. Biol. Sci. 2010, 1, 569.

[20] J. M. Tingirikari, D. Kothari, A. Goyal, Food Funct. 2014, 5, 2324.

[21] M. J. Lerman, J. Lembong, S. Muramoto, G. Gillen, J. P. Fisher, Tissue Eng. Part BRev. 2018, 24, 359.

[22] H. Wang, Y. Sun, W. Yue, Q. Kang, H. Li, D. Shen, Analyst 2018, 143, 1670.

[23] a) D. Bruen, C. Delaney, L. Florea, D. Diamond, Sensors (Basel) 2017, 17, 1866; b) R. S. Dey, R. K. Bera, C. R. Raj, Anal. Bioanal. Chem. 2013, 405, 3431.

[24] P. P. Tomanin, P. V. Cherepanov, Q. A. Besford, A. J. Christofferson, A. Amodio, C. F. McConville, I. Yarovsky, F. Caruso, F. Cavalieri, ACS Appl. Mater. Interfaces 2018, 10,42786 . 
WILEY-VCH
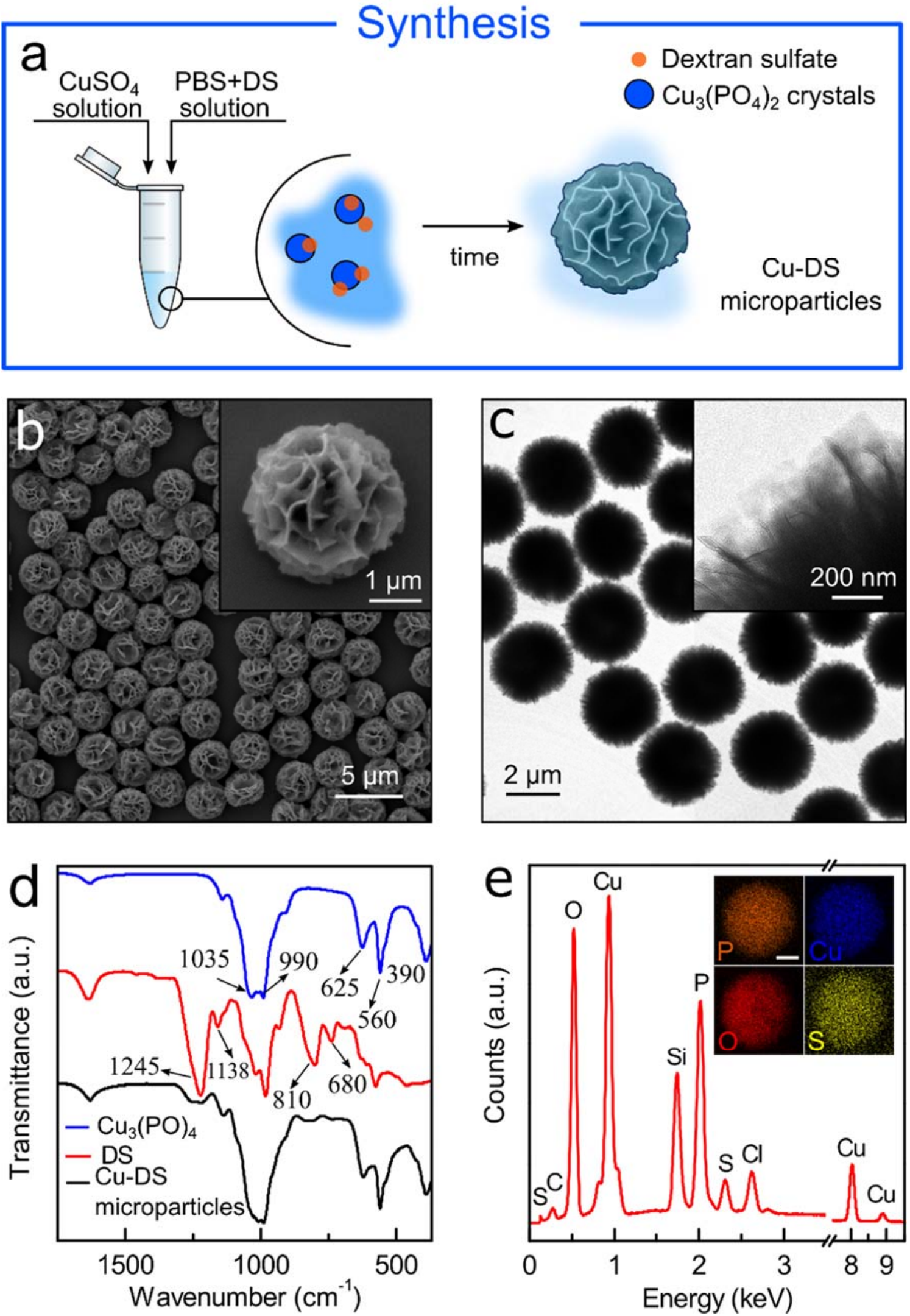

Figure 1. (a) Schematic of the preparation of Cu-DS microparticles, (b) SEM and (c) TEM images of the $\mathrm{Cu}-\mathrm{DS}$ microparticles, and respective high-magnification images (insets). [PBS] $=10 \mathrm{mM}, \mathrm{pH} 7.4,[\mathrm{DS}]=1 \mathrm{mg} \mathrm{mL}^{-1},\left[\mathrm{CuSO}_{4}\right]=120 \mathrm{mM}$, incubation time $=16 \mathrm{~h}$. (d) FTIR spectra of pure copper phosphate, DS, and hybrid Cu-DS microparticles. (e) EDX spectrum with inset showing the element mapping of the $\mathrm{Cu}-\mathrm{DS}$ microparticles. 


\section{WILEY-VCH}
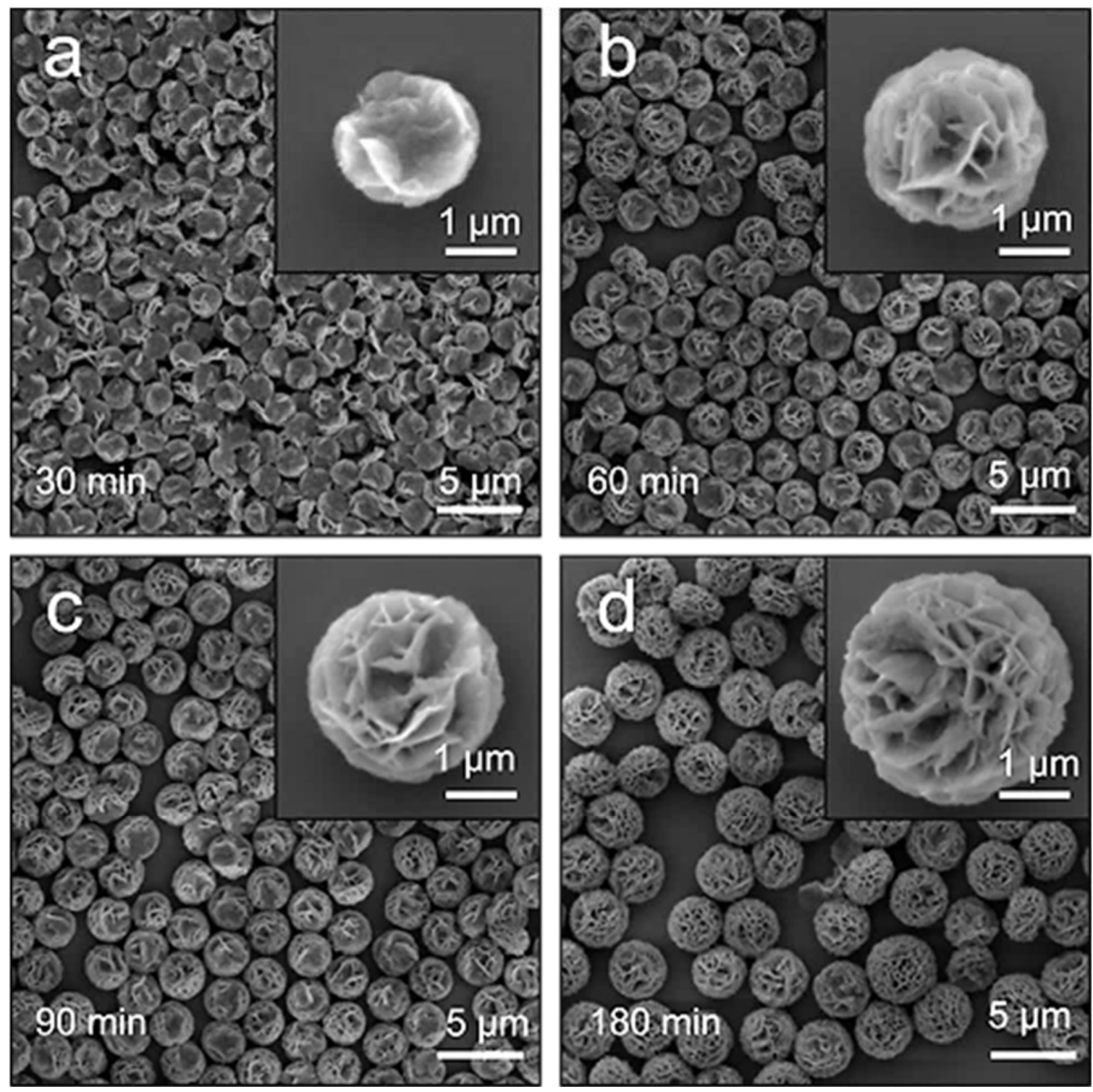

Figure 2. SEM images showing the time-dependent growth of the Cu-DS microparticles at (a) 30, (b) 60, (c) 90, and (d) 180 min of incubation measured from the start of the crystallization reaction. The $\mathrm{Cu}-\mathrm{DS}$ microparticles were prepared using DS $\left(0.83 \mathrm{mg} \mathrm{mL}^{-1}\right), \mathrm{CuSO}_{4}$ solution (20 mM), and PBS (10 mM, pH 7.4). 


\section{WILEY-VCH}
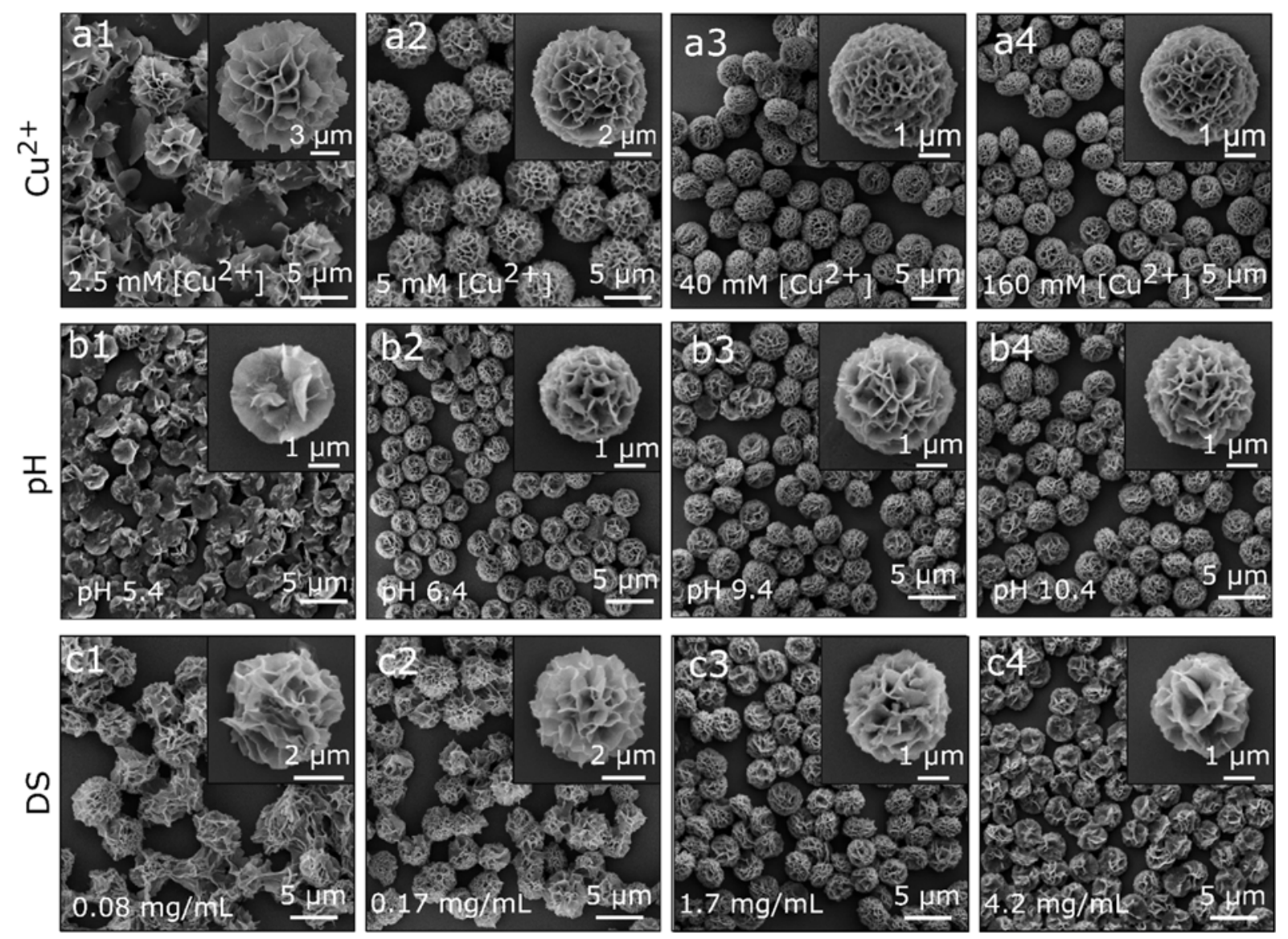

Figure 3. Low-magnification and high-magnification (insets) SEM images of Cu-DS microparticles prepared at different concentrations of $\mathrm{Cu}^{2+}$ ions: (a1) 2.5, (a2) 5, (a3) 40, and (a4) $160 \mathrm{mM}\left([\mathrm{DS}]=0.83 \mathrm{mg} \mathrm{mL}^{-1}, \mathrm{pH}=7.4,[\mathrm{PBS}]=10 \mathrm{mM}\right.$, incubation time $\left.=180 \mathrm{~min}\right)$. Low-magnification and high-magnification (insets) SEM images of Cu-DS microparticles prepared at different $\mathrm{pH}$ values: (b1) 5.4, (b2) 6.4, (b3) 9.4, and (b4) $10.4\left(\left[\mathrm{Cu}^{2+}\right]=20 \mathrm{mM}\right.$, $[D S]=0.83 \mathrm{mg} \mathrm{mL}^{-1}$, incubation time $\left.=180 \mathrm{~min}\right)$. Low-magnification and high-magnification (insets) SEM images of $\mathrm{Cu}$-DS microparticles prepared at different concentrations of DS: (c1) 0.08 , (c2) 0.17 , (c3) 1.7 , and (c4) $4.2 \mathrm{mg} \mathrm{mL}^{-1}\left(\left[\mathrm{Cu}^{2+}\right]=20 \mathrm{mM}, \mathrm{pH}=7.4\right.$, incubation time $=$ $180 \mathrm{~min})$.
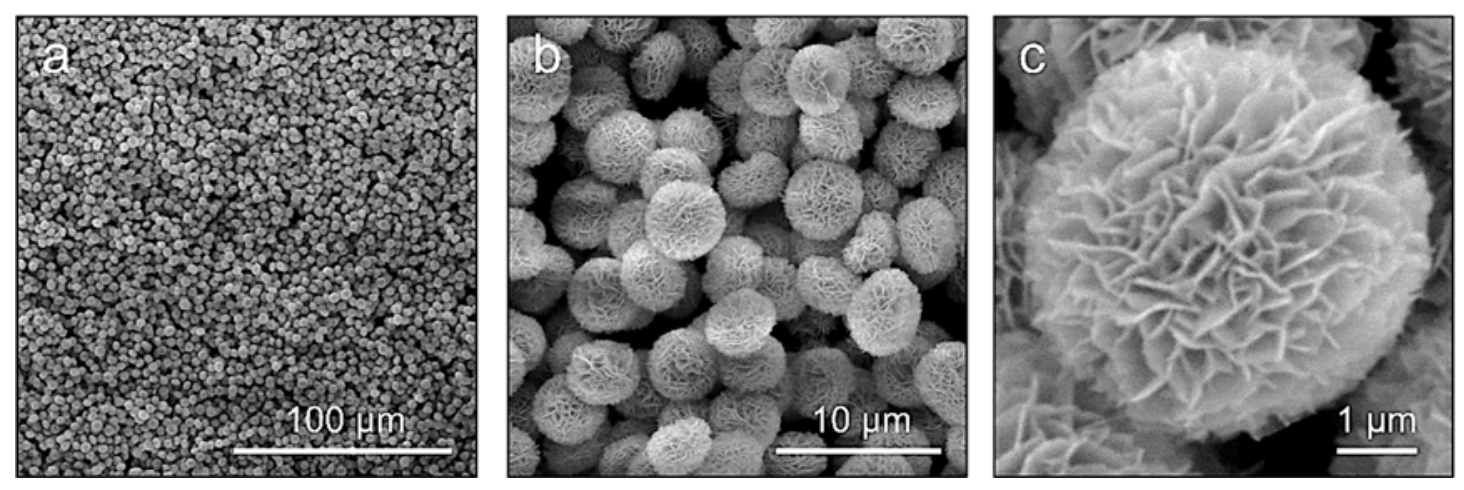

Figure 4. SEM images of Cu-DS microparticles assembled onto TC-treated PS-plates at (a) low, (b) medium, and (c) high magnifications. 

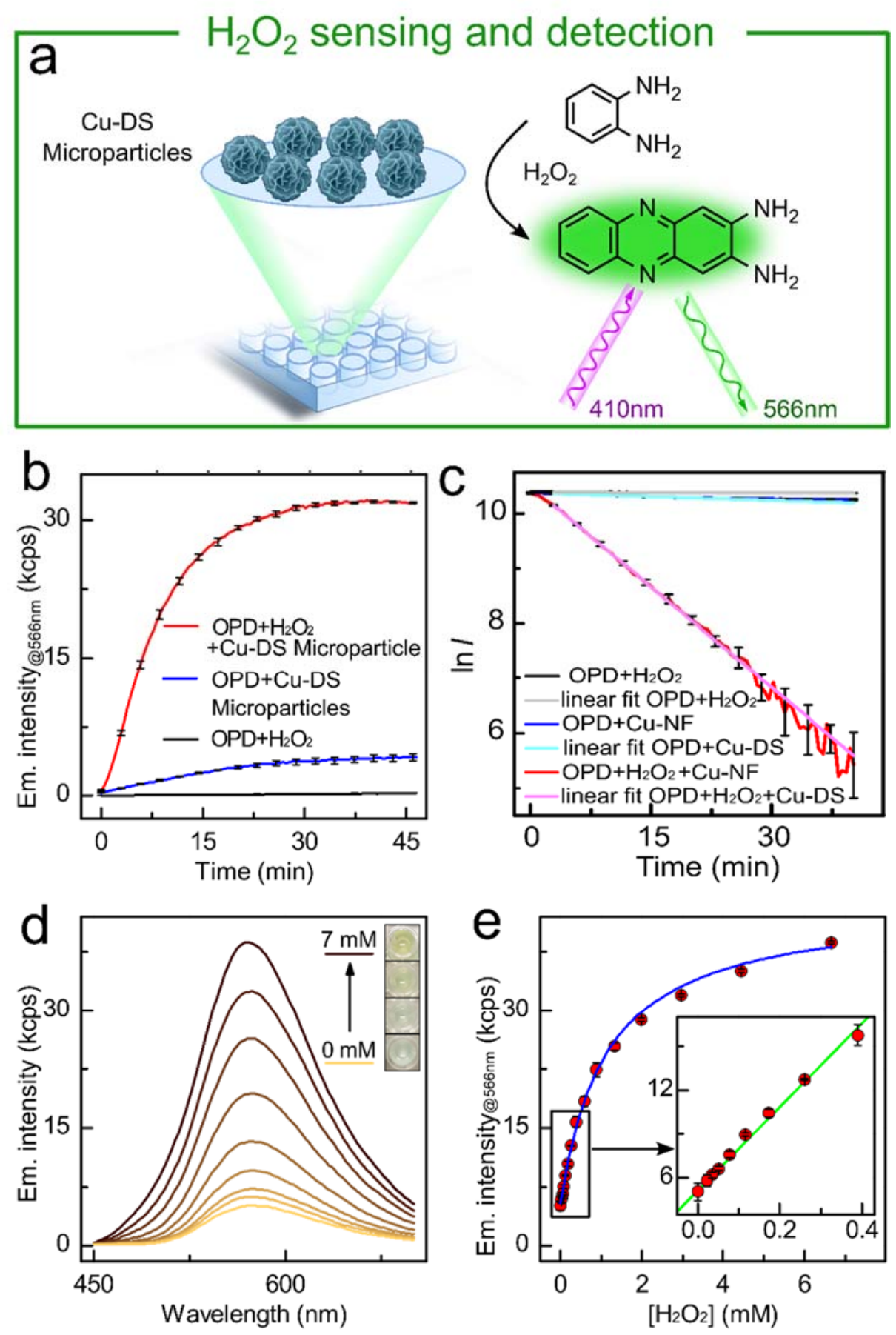

Figure 5. (a) Schematic of the Cu-DS microparticle coating platform used for the detection and sensing of hydrogen peroxide in PBS (10 mM, pH 6.5). (b) Catalytic oxidation of OPD to DAP under different conditions: in the presence of (i) $\mathrm{H}_{2} \mathrm{O}_{2}$ only, (ii) Cu-DS microparticle coating only, or (iii) $\mathrm{H}_{2} \mathrm{O}_{2}$ and Cu-DS microparticle coating - fluorescence emission intensities were recorded at $566 \mathrm{~nm}$ and the excitation wavelength was $410 \mathrm{~nm}$. (c) Pseudo-first-order plots of $\ln I$ as a function of reaction time for the reaction performed under different conditions ( $I$ is emission intensity of DAP). (d) Emission spectra and corresponding photographs (inset) of solutions incubated in the functionalized wells with the addition of $\mathrm{H}_{2} \mathrm{O}_{2}$ at varying concentrations (from 0 to $7 \mathrm{mM}$ ) in PBS buffer. (e) Fitted data plots of the variations in emission intensity as a function of $\mathrm{H}_{2} \mathrm{O}_{2}$ concentration; the inset is a magnified view of the fitted data plot at the lower $\mathrm{H}_{2} \mathrm{O}_{2}$ concentration range. 


\section{WILEY-VCH}
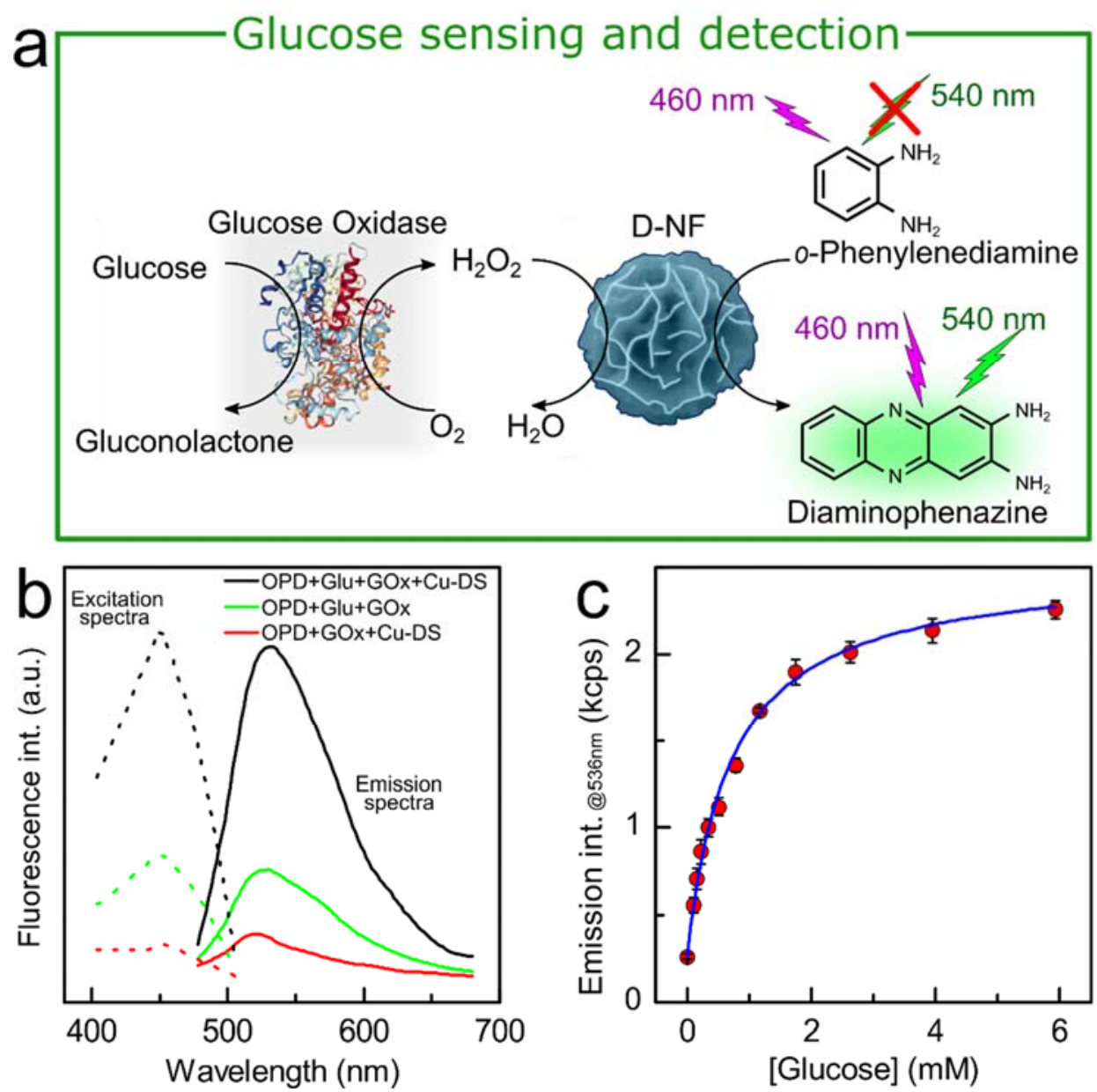

Figure 6. (a) Scheme depicting the sensing of Glu via a hybrid enzymatic/nonenzymatic cascade reaction using the engineered $\mathrm{Cu}-\mathrm{DS}$ microparticle coating platform. (b) Excitation and emission fluorescence spectra of the reaction solutions (containing varying combinations of OPD, GOx, Glu, and Cu-DS microparticles in PBS) after incubation for 120 min at $37^{\circ} \mathrm{C}$. (c) Variations in emission intensity as a function of Glu concentration. 


\section{WILEY-VCH}

\section{Table of contents}

The synthesis of hybrid porous microparticles based on copper phosphate and dextran sulfate and their assembly into macroporous coating is reported. The microparticles displayed highly uniform size, high stability at varied $\mathrm{pH}$, controlled porosity, and catalytic activity. The coatings have been employed for bioanalyte sensing and they offer high-throughput detection and sensing of $\mathrm{H}_{2} \mathrm{O}_{2}$ and glucose at physiological concentrations.

Pietro Pacchin Tomanin, Sukhvir Kaur Bhangu, Frank Caruso* and Francesca Cavalieri*

Catalytically Active Copper Phosphate-Dextran Sulfate Microparticle Coatings for Bioanalyte Sensing

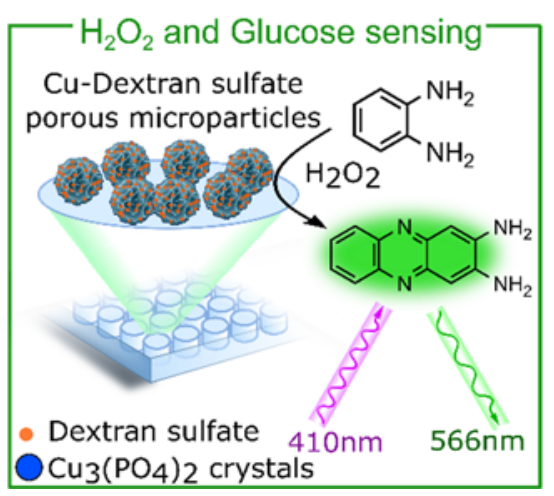


WILEY-VCH 


\section{University Library}

\section{- M M I E E R VA A gateway to Melbourne's research publications}

Minerva Access is the Institutional Repository of The University of Melbourne

Author/s:

Pacchin Tomanin, P;Bhangu, SK;Caruso, F;Cavalieri, F

Title:

Catalytically Active Copper Phosphate-Dextran Sulfate Microparticle Coatings for Bioanalyte Sensing

Date:

2020-12

Citation:

Pacchin Tomanin, P., Bhangu, S. K., Caruso, F. \& Cavalieri, F. (2020). Catalytically Active Copper Phosphate-Dextran Sulfate Microparticle Coatings for Bioanalyte Sensing. Particle and Particle Systems Characterization, 37 (12), https://doi.org/10.1002/ppsc.202000210.

Persistent Link:

http://hdl.handle.net/11343/247756 